\title{
CO Adsorption on Pd(111) at 0.5ML: A First Principles Study
}

\author{
Zahra Hooshmand, Duy Le, ${ }^{*}$ and Talat S. Rahman ${ }^{*}$ \\ Department of Physics, University of Central Florida, Orlando FL 32816-2385, USA
}

It is well-known experimentally that at $1 / 2$ monolayer (ML) coverage CO forms a $c(4 \times 2)$ phase on $\operatorname{Pd}(111)$. There is, however, a debate about whether this adsorption is at the bridge or at the hollow (FCC and HCP) sites, or at a combination of these two types of sites. Using density functional theory based calculations to evaluate the structural and vibrational properties of the $\mathrm{c}(4 \times 2)$ overlayer structure of $\mathrm{CO}$ on $\mathrm{Pd}(111)$, with all possible highly symmetric adsorption sites, we conclude that the $\mathrm{CO}$ molecules prefer to adsorb either only on the hollow (FCC or $\mathrm{HCP}$ ) sites or only at sites which are located in-between the bridge and the FCC sites and that there is no stable overlayer structure in which the molecule binds only at the bridge sites or combination of bridge and hollow sites.

Keywords: Density Functional Theory, c(4×2), CO adsorption, $\operatorname{Pd}(111)$, overlayer formation

\section{INTRODUCTION}

$\mathrm{CO}$ adsorption on the $\mathrm{Pd}(111)$ surface is an interesting topic not only because of fundamental interest but also because Pd is widely used as an industrial catalyst. Despite extensive efforts in understanding the adsorption of $\mathrm{CO}$ on $\mathrm{Pd}(111)$ with almost all available surface science techniques [1-12] and state-of-the-art theoretical and computational methods [10, 13-17], it is surprising that the overlayer structure at $1 / 2$ monolayer (ML) coverage is still not well characterized, with contradictory information coming from different experimental and theoretical works. On the other hand, adsorption structures of $\mathrm{CO}$ on $\mathrm{Pd}(111)$ at low and high coverages are well understood. For example, there is agreement that for coverages less than 1/3ML, there is no ordered structure, rather $\mathrm{CO}$ molecules are found to adsorb on three-fold hollow (TFH) sites [2, $18,19]$. Between $1 / 3 \mathrm{ML}$ and $3 / 4 \mathrm{ML}$, however, several ordered structures have been reported [4]. At $1 / 3 \mathrm{ML}$ coverage, a $(\sqrt{3} \times \sqrt{3}) \mathrm{R} 30^{\circ}$ overlayer with $\mathrm{CO}$ molecules occupying FCC hollow sites is found [1]. This site assignment is not surprising, since at such low coverages the interaction between $\mathrm{CO}$ and the metal surface is the dominant factor that determines the binding site. At $3 / 4 \mathrm{ML}$ coverage, however, intermolecular interactions become important and the overlayer structure assumes a $(2 \times 2)$ structure with (three) $\mathrm{CO}$ molecules adsorbed on the top and the FCC and HCP hollow sites. The above adsorption sites and overlayer structures of molecules have been confirmed experimentally [2] and with density functional theory (DFT) based calculations $[2,13,20]$.

At $1 / 2 \mathrm{ML}$ coverage, interactions of $\mathrm{CO}$ molecules with both the metal surface and amongst themselves (intermolecular) become equally important which makes the understanding of the overlayer structure more complex and have led to contradictory interpretations by different

\footnotetext{
*Corresponding authors. Email address: Duy.Le@ucf.edu and Talat.Rahman@ucf.edu
} 
research groups. Results from Low Energy Electron Diffraction (LEED) experiments [2, 4] are in agreement that the structure is $\mathrm{c}(4 \times 2)-2 \mathrm{CO}$. However, there is no such consensus on the nature of the binding sites. Infra-Red Reflection-Absorption Spectroscopy (IRAS) measurements propose adsorption at the bridge sites (Bridge-Bridge) [2, 3, 5], while photoelectron diffraction [6], High Resolution X-ray Photoelectron Spectroscopy (HR-XPS) and High Resolution Electron Energy Loss Spectroscopy (HR-EELS) [7] point to the involvement of only FCC and HCP (FCC-HCP) hollow sites [6, 7]. At the same time, Scanning Tunneling Microscopy (STM) study of Rose et al. [9] showed the coexistence of two islands at $1 / 2 \mathrm{ML} \mathrm{CO}$ coverage on $\mathrm{Pd}(111)$ in which binding sites assignment for each island, based on the appearance of protrusions, concluded simultaneous presence of both Bridge-Bridge and FCC-HCP occupancies. On the other hand, High Resolution Core Level Shift Spectroscopy (HRCLS) combined with DFT based calculations by Martin et al. [10] concluded that at this coverage the $\mathrm{CO}$ molecules adsorb on a mixture of FCC-Bridge and HCP-Bridge sites.

The question thus remains: is the bridge site involved in the $c(4 \times 2)-2 \mathrm{CO}$ structure on $\operatorname{Pd}(111)$ ? We address this question by carrying out first principles calculations based on DFT of the overlayer structure of $\mathrm{CO}$ on the $\mathrm{Pd}(111)$ surface at $1 / 2 \mathrm{ML}$ coverage. We show that indeed two possible combinations of adsorption sites exist: the first consists of $\mathrm{CO}$ molecules adsorbed on equal mixture of FCC and HCP hollow sites (FCC-HCP) and the second consists of $\mathrm{CO}$ molecules adsorbed on sites located between the FCC hollow and the bridge sites (FCC-Bridge Hybrid).

The paper is organized as follows: In the next section, calculational details are provided. In section 3 we discuss the results from this study in view of those from previous experimental and theoretical results.

\section{CALCULATION METHOD}

The calculations in this study include those of the system total energy, adsorbate vibrational frequencies, and simulation of STM images. Some details for each are given below.

\subsection{Total energy calculations}

We performed DFT [21, 22] calculations employing the projector-augmented wave (PAW) [23, 24], plane-wave basis set, and the supercell method as implemented in the Vienna Ab-initio Simulation Package (VASP) [25]. We described the exchange-correlation interaction of electrons by the generalized gradient approximation (GGA) in the form of Perdew-Burke-Ernzerhoff (PBE) functional [26]. We set the cutoff for kinetic energy of plane-wave expansion at $500 \mathrm{eV}$. Our supercell consists of a five layers of $\mathrm{Pd}(111)$ slab constructed with optimized lattice parameter of $3.94 \AA$, with CO molecules adsorbed on one side of the slab and a vacuum of $20 \AA$ to separate the slab and its normal periodic images. We sampled the Brillouin zone with a uniform $18 \times 18 \times 18$ and $9 \times 11 \times 1$ mesh centered at the zone center corresponding to a unit cell of bulk $\mathrm{Pd}$ and the $\mathrm{Pd}(111)$ surface (see Fig. 1), respectively. We relaxed all structures until all components of the force acting on each atom were smaller than $0.001 \mathrm{eV} / \AA$.

To estimate the role of van der Waals (vdW) interactions in determining the adsorption sites at $1 / 2 \mathrm{ML}$ coverage, calculations were also performed by using the optB88-vdW [27] functional. For testing purpose, some of the calculations were repeated with PW91 [28] functional as well. 
For all optimized structures (obtained after ionic-relaxation) the binding energy (BE) is calculated by $B E=E_{\text {slab+adsorbate }}-E_{\text {slab }}-2 \times E_{\text {adsorbate, }}$ in which $E_{\text {slab+adsorbate }}$ is the total energy of the system consisting of the $\mathrm{Pd}(111)$ slab and $\mathrm{CO}, E_{\text {slab }}$ represents the total energy of the clean $\operatorname{Pd}(111)$ slab and $E_{\text {adsorbate }}$ gives the total energy of the CO molecule in gas phase. The binding energies are then averaged over the number of $\mathrm{CO}$ molecules in the unit cell.

\subsection{Calculations of vibrational frequencies}

We calculated vibrational frequencies of the system by using the finite difference method in which atoms are displaced by $0.01 \AA$ along each direction of the three Cartesian coordinates. The forces on the atoms so generated are calculated using Hellmann-Feynman theorem. From the obtained forces, the Hessian matrix was constructed and diagonalized using the Phonopy code [29]. A scaling factor of 1.0216 was applied to obtain frequencies so that the calculated C-O stretch frequency in gas phase equals the experimental value of $2170 \mathrm{~cm}^{-1}$ [30].

\subsection{Simulation of STM images}

We simulated STM images [31] by using the Tersoff and Hamann [32, 33] method, i.e. the tunneling current at low voltage is assumed to be proportional to the local density of states (LDOS) around the Fermi level at the position of the tip apex. In our calculations, the topological STM image is approximated to be the LDOS isosurface with value of $10^{-6} \mathrm{e} / \AA^{3}$ contributed from all states in the energy range of $-0.1 \mathrm{eV}$ to $0.00 \mathrm{eV}$ (i.e. within $0.1 \mathrm{eV}$ of the Fermi level).

\section{RESULTS AND DISCUSSIONS}

\subsection{Testing the reliability of DFT functional}

Previous body of work [34-41] suggests that DFT does not always predict correctly the adsorption site for $\mathrm{CO}$ on close-packed transition metal surfaces. We tested the case of $1 / 3 \mathrm{ML}$ coverage of $\mathrm{CO}$ on $\mathrm{Pd}(111)$ because we are aware from experiments that at this coverage $\mathrm{CO}$ molecules form the $(\sqrt{3} \times \sqrt{3}) \mathrm{R} 30^{\circ}$ overlayer occupying the FCC hollow sites [2]. We considered the three high symmetry adsorption sites, i.e. the FCC and HCP hollow sites and the top site, and verified that the most preferable adsorption site is the FCC. This result implies that the PBE functional is reliable and appropriate for our model system. We also tested the case of a single $\mathrm{CO}$ on $\mathrm{Pd}(111)$ and found that $\mathrm{CO}$ prefers to bind on the FCC site and the energy difference between FCC and HCP sites is $20 \mathrm{meV}$, which is in excellent agreement with the results of Mitsui et al. [19].

\subsection{CO overlayer structure on $\operatorname{Pd}(111)$ at $1 / 2 \mathrm{ML}$}

Fig. 1 shows all surface unit cells of $\mathrm{CO}$ on $\mathrm{Pd}(111)$ at $1 / 2 \mathrm{ML}$ coverage forming $\mathrm{c}(4 \times 2)-2 \mathrm{CO}$ overlayer that we have considered in this study. After ionic relaxations (results of which are summarized in Table 1), these structures convert to either one of the following: the "FCC-HCP" structure with equal mixture of the FCC and the HCP adsorption sites (Fig. 2a) and the "FCCBridge Hybrid" structure with both $\mathrm{CO}$ molecules binding to the sites located between the FCC and bridge sites (Fig. 3a). The average binding energy of the CO molecule in the FCC-HCP and FCC-Bridge Hybrid structures are $-1.83 \mathrm{eV},-1.77 \mathrm{eV}$, respectively. The small difference in the binding energies suggests a slight preference of FCC-HCP over FCC-Bridge Hybrid (see Supplementary Material for their coordinates). We found no imaginary vibrational frequency for the obtained structures suggesting their dynamical stability. 
The results presented herein after are obtained with the PBE functional of DFT. Usage of the PW91 [28] and the optB88-vdW [27] functionals led to preference for similar binding sites as with $\mathrm{PBE}$ but with a slight difference in binding energy of $\mathrm{CO}$ molecules, as listed in Table 2.

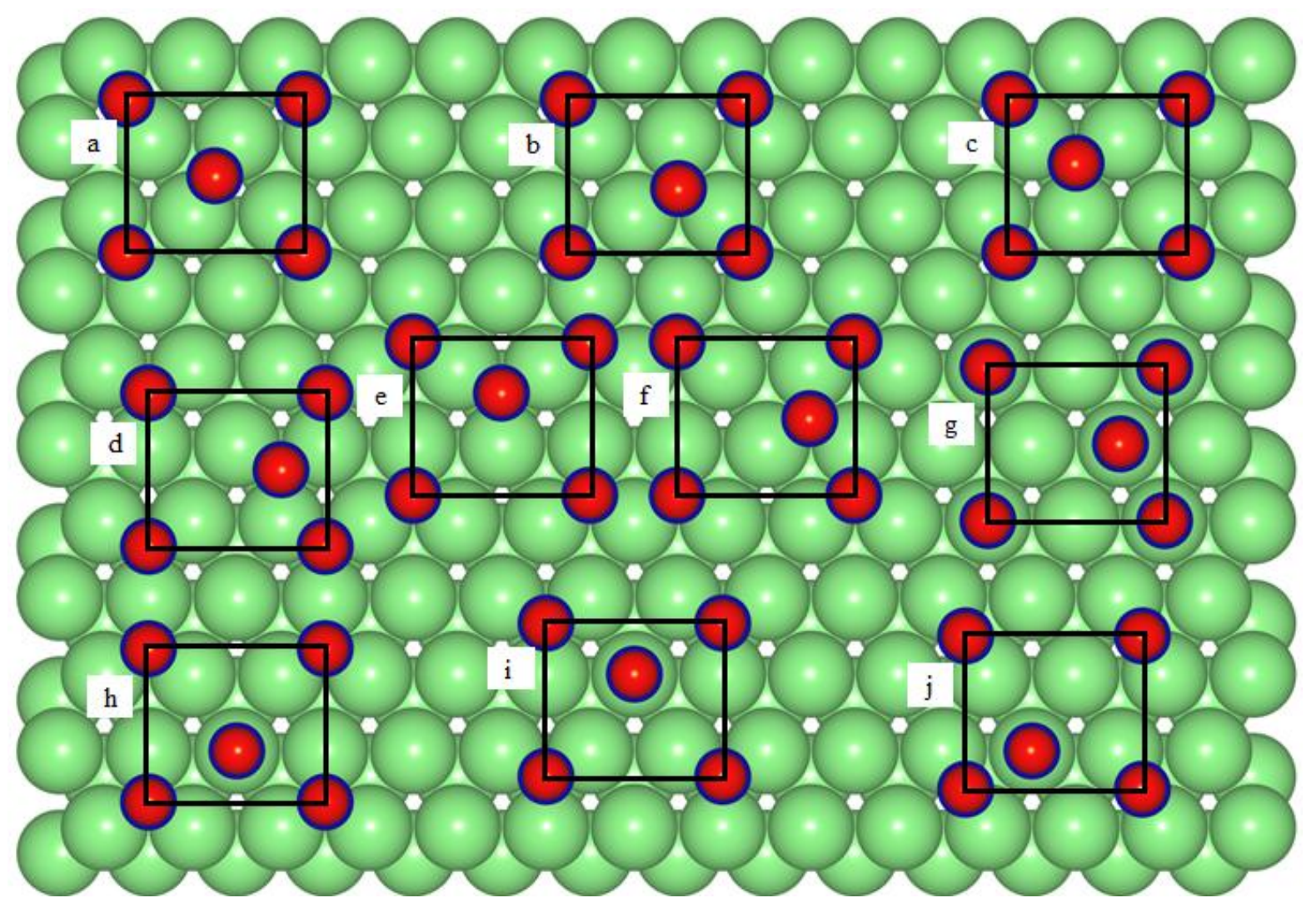

Figure 1. Considered binding sites at $1 / 2 \mathrm{ML}$ forming $\mathrm{c}(4 \times 2)-2 \mathrm{CO}$ structure as listed in Table 1. Each rectangle represents a $\mathrm{c}(4 \times 2)-2 \mathrm{CO}$ surface unit cell. The notations a-j are described in Table 1.

Table 1. The final binding sites obtained after ionic relaxation of the possible initial $\mathrm{c}(4 \times 2)-2 \mathrm{CO}$ configurations shown in Figure 1, using the PBE and optB88-vdW functionals.

\begin{tabular}{|c|c|c|}
\hline Initial Configuration & $\begin{array}{c}\text { Final Configuration } \\
\text { (PBE) }\end{array}$ & $\begin{array}{c}\text { Final Configuration } \\
\text { (optB88-vdW) }\end{array}$ \\
\hline a: Bridge-Bridge & FCC-HCP & FCC-HCP \\
\hline b: Bridge-FCC & FCC-HCP & FCC-HCP \\
\hline c: Bridge-HCP & FCC-HCP & FCC-HCP \\
\hline d: HCP-HCP & FCC-HCP & FCC-Bridge Hybrid \\
\hline e: FCC-HCP & FCC-HCP & FCC-HCP \\
\hline f: FCC-FCC & FCC-Bridge Hybrid & FCC-Bridge Hybrid \\
\hline g: TOP-TOP & FCC-Bridge Hybrid & FCC-Bridge Hybrid \\
\hline h: FCC-TOP & FCC-HCP & FCC-HCP \\
\hline i: HCP-TOP & FCC-HCP & FCC-HCP \\
\hline j: Bridge-TOP & FCC-HCP & FCC-Bridge Hybrid \\
\hline
\end{tabular}

Table 2. Calculated binding energies (in $\mathrm{eV}$ ) and $\mathrm{C}-\mathrm{O}$ stretch frequencies (in $\mathrm{cm}^{-1}$ ) on $\mathrm{Pd}(111)$ at $1 / 2 \mathrm{ML}$ obtained using different DFT functionals. 


\begin{tabular}{|c|c|c|c|}
\hline & & FCC-HCP & $\begin{array}{l}\text { FCC-Bridge } \\
\text { Hybrid }\end{array}$ \\
\hline \multirow{4}{*}{ BE (eV) } & PBE & $\begin{array}{c}-1.83 \\
-1.82^{\mathrm{a}} \\
-1.85^{\mathrm{b}}\end{array}$ & -1.77 \\
\hline & $\begin{array}{l}\text { PBE } \\
\text { with ZPE Correction (eV) }\end{array}$ & -1.78 & -1.71 \\
\hline & PW91 & $\begin{array}{c}-1.82 \\
-1.85^{\mathrm{c}} \\
-1.84^{\mathrm{d}} \\
\end{array}$ & -1.76 \\
\hline & optB88-vdW & -1.92 & -1.86 \\
\hline \multirow{3}{*}{$\begin{array}{l}\mathrm{C}-\mathrm{O} \\
\left(\mathrm{cm}^{-1}\right)\end{array}$ stretch frequency } & PBE & $\begin{array}{l}1910,1842 \\
1917,1852^{a} \\
1928,1858^{\mathrm{b}}\end{array}$ & 1923,1855 \\
\hline & PW91 & $\begin{array}{l}1914,1847 \\
1906,1850^{c}\end{array}$ & 1923,1857 \\
\hline & optB88-vdW & 1929,1859 & 1944,1874 \\
\hline
\end{tabular}

${ }^{a} \operatorname{Ref}[10]$

${ }^{\mathrm{b}} \operatorname{Ref}[14]$

${ }^{\mathrm{c}} \operatorname{Ref}[13]$

${ }^{\mathrm{d}} \operatorname{Ref}[17]$

\subsection{Discussion}

\subsubsection{FCC-HCP binding sites}
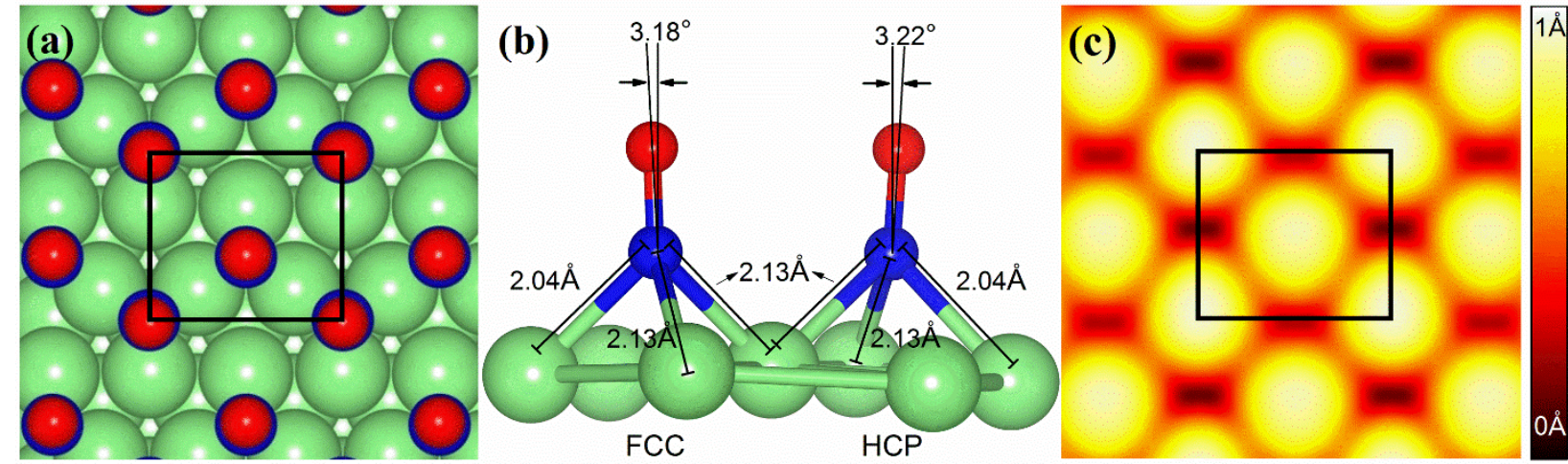

Figure 2. FCC-HCP structure: (a) Top view of structure with $\mathrm{c}(4 \times 2)-2 \mathrm{CO}$ unit cell, (b) side view showing the tilt angles of the CO molecules and C-Pd bond-lengths when adsorbed on FCC and HCP sites and (c) simulated STM image.

The FCC-HCP is one of the structures of $\mathrm{c}(4 \times 2)-2 \mathrm{CO}$ on $\mathrm{Pd}(111)$ that was suggested from analysis of experimental data $[6,9]$ and shown theoretically to be the lowest energy one for $1 / 2 \mathrm{ML}$ coverage $[10,13,14,42]$. Our ionic relaxations of several different starting structures also result in FCC-HCP as the final structure (see Table 1). The relaxed FCC-HCP structure shown in Fig. 2a displays a tilted geometry which is further elaborated in Fig. 2b. More quantitatively, we find the $\mathrm{CO}$ molecules, in fcc and hcp sites, to be tilted with angles of $3.18^{\circ}$ and $3.22^{\circ}$, 
respectively, with respect to the surface normal. In its simulated STM image (Fig. 2c), the protrusions appear in a periodic arrangement at the vertices and near the center (Fig. 2c) of a rectangular unit cell with dimension of $4.82 \times 5.57 \AA$. Because STM captures electronic properties at few angstroms away from the surface and the molecules, the tilted binding of $\mathrm{CO}$ results in appearance of protrusions adjacent to the positions of molecules as obtained by Rose et al. [9] who concluded that $\mathrm{CO}$ molecules occupy bridge sites (bridge-bridge structure) with spatially equal distance between them. However, such structural assignment was found to be unstable in our calculations. We find the average binding energy of $\mathrm{CO}$ to be $-1.83 \mathrm{eV}$, in agreement with previous theoretical results $[10,13,14]$.

\subsubsection{Bridge-Bridge binding sites}

The Bridge-Bridge geometry in which both $\mathrm{CO}$ molecules are on bridge sites is one of the suggested $\mathrm{c}(4 \times 2)-2 \mathrm{CO}$ structure [2, 3, 8, 9] (shown in Fig. 1a). This assignment was made largely because the frequency of the reported $\mathrm{CO}$ stretching mode $[2,3,8]$, in the range of 1880$2000 \mathrm{~cm}^{-1}$, was assigned to two-fold coordinated sites based on single molecule vibration frequencies [2] and the similarity of its geometric structure and STM image [9]. With force threshold of $0.01 \mathrm{eV} / \AA$, we could obtain this structure with $\mathrm{CO}$ binding energy of $-1.76 \mathrm{eV}$. This structure was also previously reported as one of relaxed structures at $1 / 2 \mathrm{ML}[10,13,14,16,42]$. However, our calculation of the vibrational frequencies for the bridge-bridge structure showed the existence of modes with imaginary frequencies, indicating the instability of the structure. Indeed, by further optimizing to a force threshold of $0.001 \mathrm{eV} / \AA, \mathrm{CO}$ molecules on the bridge sites were found to move to TFH sites, and next form the FCC-HCP structure.

\subsubsection{FCC-BRIDGE Hybrid binding sites}
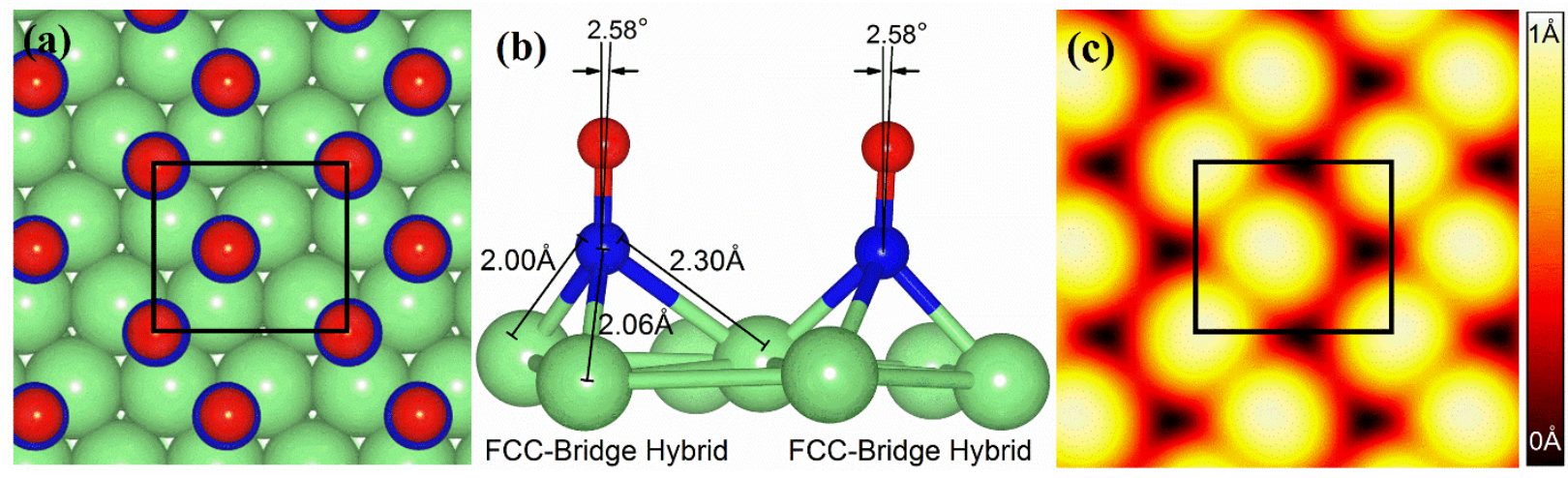

The FCC-Bridge Hybrid adsorption geometry of $\mathrm{c}(4 \times 2)-2 \mathrm{CO}$ on $\mathrm{Pd}(111)$ found in the present work has not been proposed or reported previously. This structure is similar to Bridge-Bridge discussed earlier, except that in this structure the $\mathrm{CO}$ molecules bind neither at the bridge site nor at the FCC site but between these two sites with C-Pd bond lengths of $2.00 \AA$ and $2.30 \AA$ and $2.06 \AA$. The relaxed FCC-Bridge Hybrid geometry is shown in Fig. $3 \mathrm{a}$ and $3 \mathrm{~b}$ and the $\mathrm{CO}$ binding energy of $-1.77 \mathrm{eV}$ is listed in Table 2. As in the FCC-HCP case, CO binds here tilted Figure 3. FCC-Bridge Hybrid structure: (a) Top view of structure with $\mathrm{c}(4 \times 2)-2 \mathrm{CO}$ unit cell, (b) side view with tilt angles of molecules and C-Pd bond-lengths on FCC-Bridge Hybrid sites and (c) simulated STM image of structure. 
with respect to the surface normal (Fig. 3b) with an angle of $2.58^{\circ}$ for both sites. Our simulated STM image of this structure (Fig. 3c) shows the zig-zag rows of protrusions similar to those also seen in experiments [9]. The problem is that the zig-zag rows in our calculations are apparently in the direction perpendicular to those in the experimental STM images [9] (in which the structure was assigned as FCC-HCP). Despite this discrepancy and further verifications required, the FCC-Bridge Hybrid structure reported here could potentially be the one that produces the zig-zag rows in experimental STM images. This is because the distances between the protrusions in the rows found in the experimental STM images appear to be very similar to those between the titled CO molecules in FCC-Bridge Hybrid structure proposed here. Note that our STM simulations do not take into account the effect of tip and temperature which may account for some of the differences. Note also that our calculated barrier, using Climbing Image Nudged Elastic Band (CI-NEB) [43] method, for transition from the FCC-Bridge Hybrid structure to FCC-HCP is very small $(1.3 \mathrm{meV})$ while the reverse transition barrier is $0.12 \mathrm{eV}$ (the barriers are calculated for two $\mathrm{CO}$ molecules). These values indicate that FCC-Bridge Hybrid structure may convert to FCC-HCP while the reverse transition is not favorable which is in accordance with the results of Ref [9] in which the structure with protrusions as obtained for FCC-HCP occurs more frequently. This also suggests that LEED experiment [2], performed in high temperature, was done with the FCC-HCP $\mathrm{c}(4 \times 2)$ overlayer structure.

\subsubsection{FCC-BRIDGE and HCP-BRIDGE binding sites}

FCC-Bridge and HCP-Bridge are the two structures recently suggested by Martin et al. [10] largely because at $1 / 2 \mathrm{ML}$ the XPS spectra has two distinct peaks, whose intensities display a 1:3 ratio, for the $\mathrm{Pd} 3 \mathrm{~d}_{5 / 2}$ component confirming the existence of two types of $\mathrm{Pd}$ atoms upon $\mathrm{CO}$ exposure. Combined with experimental results, their DFT calculations show the possibility of existence of four structures at $1 / 2$ ML coverage: Bridge-Bridge, FCC-HCP, FCC-Bridge and HCP-Bridge. However only Bridge-Hollow structures satisfy the 1:3 ratio observed experimentally in which $1 \mathrm{Pd}$ atom is coordinated with 2 (bridge bonded) $\mathrm{CO}$ molecules and 3 $\mathrm{Pd}$ atoms are coordinated with 1 (TFH bonded) $\mathrm{CO}$ molecule. They obtained binding energies of FCC-Bridge and HCP-Bridge structures are $-1.76 \mathrm{eV}$ and $-1.75 \mathrm{eV}$, respectively. Based on this assignment, their measured IR frequency (a strong peak at $1959 \mathrm{~cm}^{-1}$ and a weaker peak at 1864 $\mathrm{cm}^{-1}$ ) has the best agreement with their calculated vibrational frequencies for HCP-Bridge structure $\left(1957 \mathrm{~cm}^{-1}\right.$ and $\left.1862 \mathrm{~cm}^{-1}\right)$. Thus they have concluded that at $1 / 2 \mathrm{ML}$ coverage the Bridge-Hollow structures are the experimentally observed ones.

On the contrary, we find phonon instabilities for the FCC-Bridge structure obtained with a force threshold of $0.04 \mathrm{eV} / \AA ̊$ or larger $(0.05 \mathrm{eV} / \mathrm{A}$ [44] used by Martin et al. [10]). The CO molecule at bridge sites, relaxes to the HCP site to form FCC-HCP structure once the force threshold is smaller than $0.04 \mathrm{eV} / \mathrm{A}$. HCP-Bridge structure was not obtained for the above mentioned force thresholds since the $\mathrm{CO}$ molecule at the bridge site was found to move to the FCC site on ionic relaxation.

It is also worth noting that the suggested structures based on 1:3 ratio of Pd types in Martin et al. [10] are in contradiction with XPS result of Surnev et al. [7] in which a 1:1 ratio is observed between two types of $\mathrm{Pd}$ atoms (2 $\mathrm{Pd}$ atoms are coordinated with $\mathrm{CO}$ molecule adsorbed on the hollow site and 2 other $\mathrm{Pd}$ atoms are coordinated with $2 / 3 \mathrm{CO}$ molecule adsorbed on the other hollow site), confirming that FCC-HCP structure is the geometry of CO molecules at $1 / 2 \mathrm{ML}$ 
coverage on $\operatorname{Pd}(111)$. On the other hand, the shifts of $\mathrm{Pd} 3 \mathrm{~d}_{5 / 2}$ components in XPS spectra with respect to the $\mathrm{Pd}$ bulk component, realized by Martin et al. [10], are very close to the shifts observed for $(2 \times 2)-3 \mathrm{CO}$ structure at almost $3 / 4 \mathrm{ML}$ coverage obtained by Surnev et al. [7] in which a 1:3 ratio can be observed between two kinds of Pd atoms. The similarities of the shifts and the ratio between two types of surface $\mathrm{Pd}$ atoms in the two experiments suggest the possibility of $\mathrm{CO}$ coverage of Martin et al. experiment [10] to be closer to $3 / 4 \mathrm{ML}$, resulting in $(2 \times 2)-3 \mathrm{CO}$ structure, not $(4 \times 2)-2 \mathrm{CO}$ as reported. Our obtained structures $(\mathrm{FCC}-\mathrm{HCP}$ and FCCBridge Hybrid) have a ratio of 1:1 between the two types of surface Pd as obtained in Ref [7].

\subsubsection{About CO stretch vibrational frequencies}

$\mathrm{C}-\mathrm{O}$ stretch vibrational frequencies were often used to describe or identify overlayer structure and adsorption sites $[2-5,8]$ on $\operatorname{Pd}(111)$. In the $\mathrm{c}(4 \times 2)-2 \mathrm{CO}$ overlayer, because of the presence of two molecules per surface unit cell, two types of $\mathrm{CO}$ stretching modes are present: symmetric and antisymmetric, as described in Ref. [13]. We found that the corresponding frequencies for FCC-HCP are $1910 \mathrm{~cm}^{-1}$ and $1842 \mathrm{~cm}^{-1}$, respectively, and for FCC-Bridge Hybrid are $1923 \mathrm{~cm}^{-1}$ and $1855 \mathrm{~cm}^{-1}$, respectively, as summarized in Table 2. The reported experimental frequencies are $1936 \mathrm{~cm}^{-1}$ [2], $1920 \mathrm{~cm}^{-1}$ [3], $1950 \mathrm{~cm}^{-1}$ [5] and $1959 \mathrm{~cm}^{-1}$ [10]. As discussed above, our calculations show that the FCC-HCP structure is the most stable and is the only structure in full accord with experimental features. Our calculated stretch frequency of $1906 \mathrm{~cm}^{-1}$ for C-O for the FCC-HCP structure is similar to that in previous theoretical works [13, 42]. On the other hand, the calculated C-O symmetric stretch frequency of $1924 \mathrm{~cm}^{-1}$ for FCC-Bridge Hybrid structure is close to results of Ref [3]. The higher stretch frequency of this structure relative to FCC-HCP is also in line with the fact that the latter is more stable than the former.

\section{CONCLUSION}

In this work we have examined the characteristics of several possible overlayer structures of $\mathrm{CO}$ on $\operatorname{Pd}(111)$ at $1 / 2 \mathrm{ML}$ coverage to bring some consensus in the ongoing debate on the adsorption site/s. We find two structures with equal mixtures of $\mathrm{CO}$ to provide good agreement with existing experimental data: FCC-HCP and a FCC-Bridge Hybrid. More importantly, we do not find the bridge site to be the preferred one at $1 / 2 \mathrm{ML}$ coverage: the Bridge-Bridge, Bridge-TOP, FCC-Bridge and HCP-Bridge structures are found to relax to FCC-HCP. Additionally, the FCCFCC and TOP-TOP configurations relax into a new structure which we call FCC-Bridge Hybrid. Our calculated STM image of the FCC-HCP structure is in good agreement with one of the experimental STM images [9] which we believe was incorrectly assigned previously to the structure in which both $\mathrm{CO}$ molecules bind to the bridge sites. Our proposed FCC-Bridge Hybrid structure is very interesting as it produces zig-zag STM images resembling those seen in experimental STM images albeit not in full agreement with the orientation of those in the experiments. Furthermore, the transition barrier for FCC-Bridge Hybrid to FCC-HCP is found to be small and the reverse barrier to be relatively large, pointing to the FCC-HCP as the most stable structure. This conclusion is also in qualitative agreement with experiments according to which the FCC-HCP structure is found more frequently in the STM images. We also found that inclusion of $\mathrm{vdW}$ interactions in the calculations did not have significant effect on our conclusions. 


\section{ACKNOWLEDGEMENT}

The calculations were performed on STOKES high performance facility at the University of Central Florida and XSEDE computational resources. This work was partially supported by NSF grant CHE-1310327.

\section{REFERENCES}

[1] H. Conrad, G. Ertl, J. Koch, E.E. Latta, Surf. Sci., 43 (1974) 462-480.

[2] A.M. Bradshaw, F.M. Hoffmann, Surf. Sci., 72 (1978) 513-535.

[3] F.M. Hoffmann, Surf. Sci. Rep., 3 (1983) 107-192.

[4] M. Tüshaus, W. Berndt, H. Conrad, A.M. Bradshaw, B. Persson, Appl. Phys. A, 51 (1990) 91-98.

[5] W.K. Kuhn, J. Szanyi, D.W. Goodman, Surf. Sci., 274 (1992) L611.

[6] T. Gießel, O. Schaff, C.J. Hirschmugl, V. Fernandez, K.M. Schindler, A. Theobald, S. Bao, R. Lindsay, W. Berndt, A.M. Bradshaw, C. Baddeley, A.F. Lee, R.M. Lambert, D.P. Woodruff, Surf. Sci., 406 (1998) 90102.

[7] S. Surnev, M. Sock, M.G. Ramsey, F.P. Netzer, M. Wiklund, M. Borg, J.N. Andersen, Surf. Sci., 470 (2000) 171-185.

[8] E. Ozensoy, D.C. Meier, D.W. Goodman, J. Phys. Chem. B, 106 (2002) 9367-9371.

[9] M.K. Rose, T. Mitsui, J. Dunphy, A. Borg, D.F. Ogletree, M. Salmeron, P. Sautet, Surf. Sci., 512 (2002) 48-60.

[10] N.M. Martin, M. Van den Bossche, H. Grönbeck, C. Hakanoglu, F. Zhang, T. Li, J. Gustafson, J.F. Weaver, E. Lundgren, J. Phys. Chem. C, 118 (2014) 1118-1128.

[11] R. Miranda, K. Wandelt, D. Rieger, R.D. Schnell, Surface Science, 139 (1984) 430-442.

[12] G. Odörfer, E.W. Plummer, H.J. Freund, H. Kuhlenbeck, M. Neumann, Surface Science, 198 (1988) 331-359.

[13] D. Loffreda, D. Simon, P. Sautet, Surf. Sci., 425 (1999) 68-80.

[14] K. Honkala, P. Pirilä, K. Laasonen, Surf. Sci., 489 (2001) 72-82.

[15] R. Chen, Z. Chen, B. Ma, X. Hao, N. Kapur, J. Hyun, K. Cho, B. Shan, Comput. Theor. Chem., 987 (2012) 77-83.

[16] C. Fan, W.-D. Xiao, Comput. Theor. Chem., 1004 (2013) 22-30.

[17] Z. Duan, G. Henkelman, ACS Catal., 4 (2014) 3435-3443.

[18] P. Sautet, M.K. Rose, J.C. Dunphy, S. Behler, M. Salmeron, Surf. Sci., 453 (2000) 25-31.

[19] T. Mitsui, M.K. Rose, E. Fomin, D.F. Ogletree, M. Salmeron, Phys. Rev. Lett., 94 (2005) 036101.

[20] I. Bakó, R. Schennach, G. Pálinkás, J. Phys.: Conf. Ser, 100 (2008) 052067.

[21] P. Hohenberg, W. Kohn, Phys. Rev., 136 (1964) B864-B871.

[22] W. Kohn, L.J. Sham, Phys. Rev., 140 (1965) A1133-A1138.

[23] P.E. Blöchl, Phys. Rev. B, 50 (1994) 17953-17979.

[24] G. Kresse, D. Joubert, Phys. Rev. B, 59 (1999) 1758-1775.

[25] G. Kresse, J. Furthmuller, Phys. Rev. B, 54 (1996) 11169-11186.

[26] J.P. Perdew, K. Burke, M. Ernzerhof, Phys. Rev. Lett., 77 (1996) 3865-3868.

[27] J. Klimeš, D.R. Bowler, A. Michaelides, Phys. Rev. B, 83 (2011) 195131.

[28] J.P. Perdew, Y. Wang, Phys. Rev. B, 45 (1992) 13244-13249.

[29] A. Togo, L. Chaput, I. Tanaka, G. Hug, Phys. Rev. B, 81 (2010) 174301.

[30] K. Nakamoto, Infrared and Raman Spectra of Inorganic and Coordination Compounds, 4th ed., Wiley, New York, 1986.

[31] D. Tománek, S.G. Louie, Phys. Rev. B, 37 (1988) 8327-8336.

[32] J. Tersoff, D.R. Hamann, Phys. Rev. Lett., 50 (1983) 1998-2001. 
[33] J. Tersoff, D.R. Hamann, Phys. Rev. B, 31 (1985) 805-813.

[34] P.J. Feibelman, B. Hammer, J.K. Nørskov, F. Wagner, M. Scheffler, R. Stumpf, R. Watwe, J. Dumesic, J. Phys. Chem. B, 105 (2000) 4018-4025.

[35] M. Lynch, P. Hu, Surf. Sci., 458 (2000) 1-14.

[36] G. Kresse, A. Gil, P. Sautet, Phys. Rev. B, 68 (2003) 073401.

[37] A. Gil, A. Clotet, J.M. Ricart, G. Kresse, M. Garcia-Hernández, N. Rösch, P. Saute, Surf. Sci., 530 (2003) 71-87.

[38] G. Marek, E. Andreas, H. Jürgen, J. Phys.: Condens. Matter, 16 (2004) 1141.

[39] S.E. Mason, I. Grinberg, A.M. Rappe, Phys. Rev. B, 69 (2004) 161401.

[40] M. Gajdoš, J. Hafner, Surf. Sci., 590 (2005) 117-126.

[41] M. Neef, K. Doll, Surf. Sci., 600 (2006) 1085-1092.

[42] X. Ma, A. Genest, L. Spanu, N. Rösch, Comput. Theor. Chem., 1069 (2015) 147-154.

[43] G. Henkelman, B.P. Uberuaga, H. Jonsson, J. Chem. Phys., 113 (2000) 9901-9904.

[44] M. Van den Bossche, Private Email Communication, (2015). 
GRAPHICAL ABSTRACT

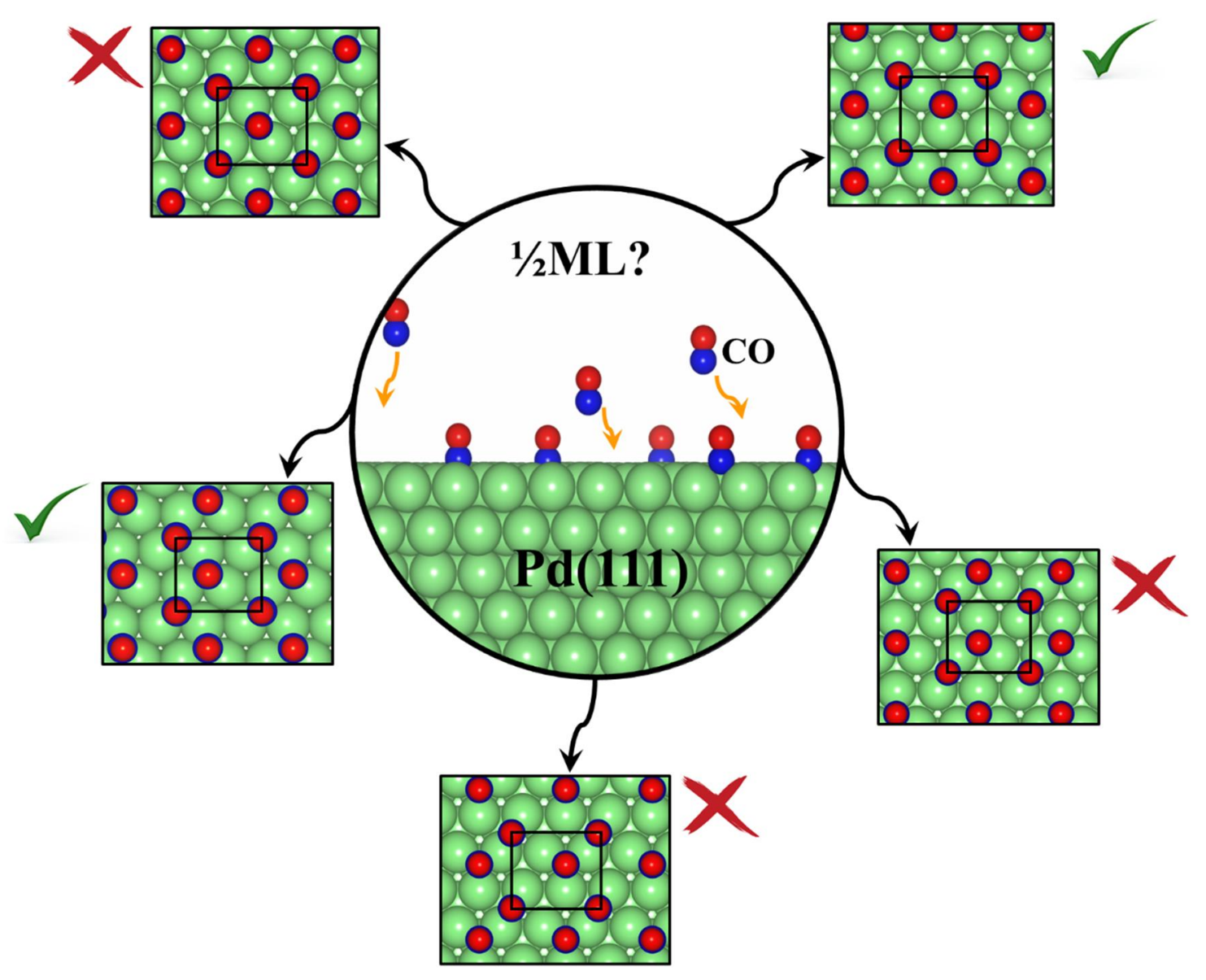

